\author{
G.K. Kurmantayeva ${ }^{1 *}$, A.B. Marchenko ${ }^{1}$, S.A. Ivasenko ${ }^{1}$, \\ R.B. Seidakhmetova ${ }^{2}$, M.K. Smagulov ${ }^{2}$, G.A. Atazhanova ${ }^{1}$ \\ ${ }^{1}$ Karaganda Medical University, Karaganda, Kazakhstan; \\ ${ }^{2}$ Karagandy University of the name of academician E.A. Buketov, Karaganda, Kazakhstan \\ *Corresponding author: kurmantaeva@qmu.kz
}

\title{
Chemical composition and biological activity of essential oil of Nepeta pannonica
}

\begin{abstract}
Search for new sources of biologically active substances from plants of local flora is a promising area of modern phytochemical science. The article examines the composition of essential oil samples obtained from Nepeta pannonica, growing in the Karaganda region with the use of gas-chromatography-mass spectrometry method. The differences in the chemical composition of the oil depending by the plant organs have been identified. The main component of essential oil is nepetalactone. For the analysis, a unified method for determining the component composition of essential oils, as well as an Agilent Technologies 7890A chromatograph system with a 5975C inert MSD mass spectrometric detector were used. According to the data, the following substances were identified in the essential oils of the plant - 1,8-cineole, nepetalactone, germacrene D, screening of essential oil of Nepeta pannonica for antimicrobial and analgesic activity.
\end{abstract}

Keywords: Nepeta pannonica, essential oil, gas-chromatography-mass spectrometry, chemical composition, antimicrobial and analgesic activity.

\section{Introduction}

Genus Nepeta L. (family Lamiaceae) comprises of 279 species; among them in Kazakhstan there are 16 species [1]. Many species belonging to the genus Nepeta have traditionally been used as biologically active agents for the treatment of many diseases. Pharmacological effects of plants include antimicrobial, antioxidant, anti-inflammatory, sedative, cholesterol-lowering, anti-asthmatic, diuretic, sweating, antipyretic, glycogenic, and other properties [2-9].

All of these effects are related to a certain chemical composition. Plants of the genus Nepeta contain unsaturated lactones called non-tetolactones. Depending on the content of this compound, genus Nepeta species can be divided into two groups, one containing nepetolactone (and isomers thereof) with a relatively high content and a second group with low nepetalactone content and major components such as 1,8-cyneol, $\beta$-caryophyllene, caryophyllene oxide, $\beta$-farnesene, $\alpha$-citral, $\beta$-citronellol [10-11].

Essential oil from Nepeta species is characterized by presence of sesquiterpene lactones and their derivates, such as germacrenes, caryophyllenes, caryophyllene oxide [2, 10-11]. Many substances contained in the essential oil of Nepeta have pronounced biological activity [12-14].

The prospects for the use of Nepeta set us the task of studying the chemical composition of the essential oil of Nepeta pannonica L., growing in the Central Kazakhstan, and determining its biological activity.

\section{Experimental}

N. pannonica raw material was collected in June, 2020; phase flowering, Spassky hills, Abay district of the Karaganda region (N 49 32'19"; E 7316 $33^{\prime \prime}$ ). Raw material was dried according to plant drying rules.

Essential oils of aboveground parts (leaves, shoots and inflorescences) were extracted by hydrodistillation method for 3 hours using a Clevenger apparatus.

To study the composition of the essential oil, a chromatograph-mass spectrometry method was used with the help of an Agilent Technologies 7890A Gas Chromatograph with a quadrupole mass spectrometer MSD $5975 \mathrm{C}$ as a detector. The capillary column HP-5MS had a size of $30 \mathrm{~m}$ x $0.25 \mathrm{~mm}$ (film thickness 0.25 $\mu \mathrm{m})$. Evaporator temperature was $230^{\circ} \mathrm{C}$. The gas chromatographic column was kept at $40{ }^{\circ} \mathrm{C}$ for 10 minutes; with temperature programming up to $240{ }^{\circ} \mathrm{C}$ at a temperature change rate of $2{ }^{\circ} \mathrm{C} / \mathrm{min}$, and then held in isothermal mode for 20 minutes. Sample injection mode was flow division 100:1. Sample volume was $0.2 \mu \mathrm{l}$. The conditions for recording mass spectra are $70 \mathrm{eV}$, the mass range is $\mathrm{m} / \mathrm{z} 10-360$. For data processing, the MSD ChemStation software, supplied by Agilent Technologies, was used in combination with AMDIS 32 and NIST 2017. 
The analgesic activity of the essential oil of $N$. pannonica was studied on an experimental model of chemical irritation of the peritoneum, induced by the introduction of acetic acid in white barren mice. The crusts were induced by intra peritoneal administration of $0.75 \%$ aqueous acetic acid solution at a dose of $1 \mathrm{ml}$ per $100 \mathrm{~g}$ of animal body weight. The essential oil was administered by intra-gastric for 30 minutes prior to administration of acetic acid. The number of crusts was calculated in 20 minutes after intra-peritoneal administration of acetic acid for 30 minutes. An essential oil in the form of starch mucus was administered by intra-gastric at a dose of $25 \mathrm{mg} / \mathrm{kg}$ by means of a special metal probe for 30 minutes prior to administration of acetic acid. A decrease in the amount of crusts in animals compared to the control group served as an indicator of the analgesic activity of the test substances. As a comparative preparation, sodium diclophenac was used in its effective dose of $8 \mathrm{mg} / \mathrm{kg}$ (YeD50 = $8 \mathrm{mg} / \mathrm{kg}$ ). Control animals received an equivalent amount of starch mucus. Analgesic activity was expressed as a percentage of the reduction in the number of acetic crusts in experimental rats compared to controls [15].

Antimicrobial activity of a sample of essential oil of $N$. pannonica was studied on reference test microorganisms: Staphylococcus aureus ATCC 6538, Bacillus subtilis ATCC 6633, Escherichia coli ATCC 25922, Pseudomonas aeruginosa ATCC 27853 and to yeast fungus Candida albicans ATCC 10231 method agar diffusion (holes). Comparative preparations are benzyl penicillin sodium salt, gentamicine for bacteria and nystatin for Candida albicans. For the study, pure cultures of test strains were taken, which were previously grown on a liquid medium pH $7.3 \pm 0.2$ at a temperature from 30 to $37^{\circ} \mathrm{C}$; for $24-48$ hours on a beveled meat-peptone agar.

A standard bacterial suspension was prepared by diluting a 1:1000 culture in sterile $0.9 \%$ sodium chloride isotonic solution. The corresponding bacterial suspension was added $1.0 \mathrm{ml}$ each to dishes with appropriate elective, nutritional media for the test strains under study and seeded by a "continuous lawn" method. After drying, $6.0 \mathrm{~mm}$ wells were formed on the surface of the agar, into which $20 \mu 1$ of the test sample was added. In the control, water for injection was used to dilute samples in equivalent amounts. The crops were incubated at $370{ }^{\circ} \mathrm{C}$ for 24 hours for the bacterium and at $300{ }^{\circ} \mathrm{C}$ for 48 hours for the yeast fungus Candida albicans $[15,16]$.

The antimicrobial activity of the sample was estimated by the diameter of the growth delay zones of test strains (mm) around the hole: i) the absence of a growth delay zone - the test culture is not sensitive to this concentration of the sample; ii) diameter of growth delay zones is less than $10 \mathrm{~mm}$ and continuous growth in the dish was evaluated as an absence of antibacterial activity; iii) $10-15 \mathrm{~mm}$ - weak activity; iv) 15 $20 \mathrm{~mm}$ - moderately expressed activity; v) over $20 \mathrm{~mm}$ - pronounced activity. The essential oil of $N$. pannonica was tested in three parallel runs.

Statistical processing of the results was carried out using the software package Statistica 8.0. Differences at the achieved significance level $\mathrm{p}<0.05$ were considered reliable.

\section{Results and Discussion}

Earlier, in the composition of the essential oil of $N$. pannonica, 36 components were identified, more of them from light fractions of monoterpenes and their oxidized forms. The major components in the essential are 1,8-cineole and $\gamma$-cadinene. The amount of eucalyptol was $28.9 \%$, nepetalactone was $14.3 \%$ [14].

Other authors investigated the above-ground part of $N$. pannonica, which was collected in the Aksai gorge of the Transili Alatau (Almaty region) in 2000. The yield of essential oil was $0.2 \%$. The essential oil composition was examined by chromatograph-mass spectrometry. It was identified 92 components; among them nepetalactone $-41.5 \%, 1,8$-cineole $-12.2 \%$, germacrene $\mathrm{D}-6.3 \%$, caryophyllene oxide $5.2 \%$, pulegon $-2.9 \%$, $\alpha$-terpineol $-1.6 \%$, and $\beta$-terpineol $-1.0 \%[15]$.

The essential oil that we have isolated from $N$. pannonica is a light yellow moving liquid with a pleasant smell. The yield of essential oil is $0.35 \%$ (Tab. 1).

In the essential oil isolated from the aboveground part of $N$. pannonica, 50 components were identified by the GC-MS method, the main ones being 1,8-cineole (11.77\%) and (4aR,7S,7aS)-nepetalactone $(18.75 \%)$. The main sesquiterpenoids are represented by caryophyllene $(3.53 \%)$ and germacrene D $(5.21 \%)$. We have determined the component composition of essential oils depending on the plant organs (Tab. 2). 
Chemical compositions of essential oil of Nepeta pannonica

\begin{tabular}{|c|c|c|c|}
\hline No. & $\begin{array}{c}\mathrm{RT}, \\
\text { minutes }\end{array}$ & Component & $\begin{array}{l}\text { Amount of component, in \% } \\
\text { from whole essential oil }\end{array}$ \\
\hline 1 & 10.736 & $\alpha$-pinene & 1.19 \\
\hline 2 & 12.208 & cis-sabinene & 0.66 \\
\hline 3 & 12.280 & $\gamma$-pinene & 1.03 \\
\hline 4 & 12.533 & 1-octen-3-ol & 0.99 \\
\hline 5 & 12.894 & $\beta$-myrcene & 0.77 \\
\hline 6 & 14.272 & 1,8 -cineole & 11.77 \\
\hline 7 & 14.856 & $\beta$-pinene & 0.78 \\
\hline 8 & 15.174 & $\gamma$-terpinene & 1.77 \\
\hline 9 & 15.462 & trans-4-thujanol & 1.11 \\
\hline 10 & 18.500 & Pyrocarbon & 0.23 \\
\hline 11 & 18.637 & cis-sabinene & 0.58 \\
\hline 12 & 18.947 & terpinen-4-ol & 0.41 \\
\hline 13 & 19.366 & $\alpha$-terpineol & 0.96 \\
\hline 14 & 19.532 & (1R)-(-)-myrtenal & 0.15 \\
\hline 15 & 20.686 & cis-3-hexenyl isovalerate & 0.45 \\
\hline 16 & 22.194 & $\begin{array}{l}\text { 2H-1-benzopyran, 3,4,4a,5,6,8a-hexahydro-2,5,5,8a-tetramethyl-(2“ } \alpha ”, \\
4 \mathrm{a} \text { “' } \alpha ”, 8 \mathrm{a} \text { “' } \alpha ”)\end{array}$ & 0.15 \\
\hline 17 & 22.367 & $n$-cymene-2-ol & 0.04 \\
\hline 18 & 22.865 & non-identified & \\
\hline 19 & 24.200 & (4aS,7S,7aR)-nepetalactone & 2.41 \\
\hline 20 & 24.575 & Copaene & 0.49 \\
\hline 21 & 24.835 & $(-)-\beta$-burbonene & 3.02 \\
\hline 22 & 25.160 & (4aR,7S,7aS)-nepetalactone & 18.75 \\
\hline 23 & 25.484 & 1H-cycloprop[e]azulene & 0.21 \\
\hline 24 & 25.593 & naphthalene, 1,2,3,4-tetrahydro-1,6,8-trimethyl & 0.12 \\
\hline 25 & 25.766 & caryophyllene & 3.53 \\
\hline 26 & 25.975 & $\alpha$-copaene-4-ol & 0.33 \\
\hline 27 & 26.358 & isogermacrane D & 0.31 \\
\hline 28 & 26.603 & Humulene & 1.40 \\
\hline 29 & 26.920 & $\beta$-cubbenen & 0.57 \\
\hline 30 & 27.317 & germacrene D & 5.21 \\
\hline 31 & 27.663 & bicyclogermacrene & 0.63 \\
\hline 32 & 27.880 & $\beta$-bisabolene & 0.56 \\
\hline 33 & 28.277 & 1(10),4-cadinadien & 0.61 \\
\hline 34 & 29.200 & epoxy daristolene & 0.72 \\
\hline 35 & 29.222 & (-)-norburbonone & 0.31 \\
\hline 36 & 29.374 & 3-hexene-1-ol, benzoate & 0.34 \\
\hline 37 & 29.763 & caryophyllene oxide & 2.49 \\
\hline 38 & 29.987 & salvial-4(14)-en-1-one & 0.24 \\
\hline 39 & 30.355 & humulene epoxide & 0.63 \\
\hline 40 & 31.055 & Cadinol & 0.19 \\
\hline 41 & 31.163 & trans-chrysantemal & 0.17 \\
\hline 42 & 31.351 & $\alpha-$ cadinol & 0.37 \\
\hline 43 & 31.726 & muurola-4(10)-dien-1-ol & 0.27 \\
\hline 44 & 32.065 & $\gamma$-muurolene & 0.29 \\
\hline 45 & 32.180 & Geneikozan & 0.08 \\
\hline 46 & 40.160 & Dokozan & 0.16 \\
\hline 47 & 41.950 & Hexakozan & 0.14 \\
\hline 48 & 43.660 & Tetrakozan & 0.15 \\
\hline 49 & 45.312 & pentakozan & 0.18 \\
\hline 50 & 47.181 & tetratracontane & 0.07 \\
\hline
\end{tabular}


The main components of essential oil of Nepeta pannonica depending on the plant organs

\begin{tabular}{|c|l|c|c|}
\hline No. & \multicolumn{1}{|c|}{ Component } & $\begin{array}{c}\text { Content of components in \% of } \\
\text { whole oil in inflorescences }\end{array}$ & $\begin{array}{c}\text { Content of components in \% of } \\
\text { whole oil in leaves and stems }\end{array}$ \\
\hline 1 & 1,8 -cyneol & 2.88 & 13.00 \\
\hline 2 & $(+)-(4 a S, 7 S, 7 a R)$-nepetalactone & 3.91 & 1.38 \\
\hline 3 & $(-)-\beta-$ burbonene & 3.09 & 3.87 \\
\hline 4 & $(4 a R, 7 S, 7 a S)$-nepetalactone & 46.55 & 18.0 \\
\hline 5 & Caryophyllene & 5.49 & 5.23 \\
\hline 6 & germacrene D & 10.69 & 6.95 \\
\hline 7 & Humulene & 2.24 & 2.23 \\
\hline 8 & caryophyllene oxide & 2.97 & 11.0 \\
\hline
\end{tabular}

According to GC-MS, the following substances are determined in essential oils of plant flowers (4aR,7S,7aS)-nepetalactone (46.55\%), germacrene D $(10.69 \%)$. The essential oils of plant leaves contained (4aR,7S,7aS)-nepetalactone (18.0), 1.8-cyneol (13\%), caryophyllene $(5.23 \%)$ and its oxide (11\%), germacrene D $(6.95 \%)$.

The results of the analysis of the antimicrobial activity of $N$. pannonica essential oil sample by diffusion into agar are shown in Tables 3, 4 .

Table 3

Antimicrobial activity of Nepeta pannonica essential oil

\begin{tabular}{|l|c|c|c|c|c|}
\hline \multicolumn{1}{|c|}{ Testing sample } & $\begin{array}{c}\text { Staphylococcus } \\
\text { aureus } \\
\text { ATCC } 6538\end{array}$ & $\begin{array}{c}\text { Bacillus } \\
\text { subtilis } \\
\text { ATCC } 6633\end{array}$ & $\begin{array}{c}\text { Escherichia } \\
\text { coli } \\
\text { ATCC 25922 }\end{array}$ & $\begin{array}{c}\text { Pseudomonas } \\
\text { aeruginosa } \\
\text { ATCC 27853 }\end{array}$ & $\begin{array}{c}\text { Candida } \\
\text { albicans ATCC } \\
10231\end{array}$ \\
\hline $\begin{array}{l}\text { Essential oil of } \\
\text { N. pannonica }\end{array}$ & $18 \pm 0.1^{*}$ & $16 \pm 0.1^{*}$ & $15 \pm 0.2$ & - & $15 \pm 0.1^{*}$ \\
\hline $\begin{array}{l}\text { Benzylpenicillin } \\
\text { sodium salt }\end{array}$ & $16 \pm 0.1$ & $14 \pm 0.1$ & $15 \pm 0.1$ & - & - \\
\hline Gentamicin & $24 \pm 0.1$ & $21 \pm 0.2$ & $26 \pm 0.1$ & $27 \pm 0.1$ & - \\
\hline Nystatin & - & - & - & & $21 \pm 0.2$ \\
\hline
\end{tabular}

Note: validity of differences $\mathrm{p}<0.05$ compared to comparison group.

Table 4

The screening of Nepeta pannonica essential oil for analgesic activity

\begin{tabular}{|l|c|c|c|c|}
\hline \multicolumn{1}{|c|}{ Testing sample } & Doze, $\mathrm{mg} / \mathrm{kg}$ & Number of crusts & \% to control & Analgesic activity \\
\hline Control & - & $105,4 \pm 11,8$ & 100 & - \\
\hline Diclofenac sodium & 8 & $53,6 \pm 10,5$ & 50.9 & 49.1 \\
\hline Essential oil of $N$. pannonica & 25 & $49,2 \pm 10,8^{*}$ & 46.7 & 53.3 \\
\hline
\end{tabular}

Note: validity of differences $\mathrm{p}<0.05$ compared to comparison group.

An antimicrobial study found that an essential oil sample exhibited moderate antimicrobial activity against the gram-positive test strains Staphylococcus aureus ATCC 6538 and Bacillus subtilis ATCC 6633, and also illustrated poor antibacterial activity against the gram-negative test strain Escherichia coli ATCC 25922. N. pannonica essential oil shows antifungal activity against yeast fungus Candida albicans ATCC 10231.

As a result of the study, it was determined that a sample of essential oil of $N$. pannonica at a dose of $25 \mathrm{mg} / \mathrm{g}$ demonstrated analgesic activity in a model of chemical irritation of the peritoneum, illustrating a significant decrease in the development of experimental bark in rats by $53.3 \%$, respectively, in comparison with the control. 


\section{Conclusions}

The work presented the result of study of $N$. pannonica essential oil, extracted by hydro-distillation method. The essential oil is a light yellow moving liquid with a pleasant odor; the yield was $0.35 \%$. For the first time, the GC-MS method determined the component composition of $N$. pannonica growing in the Karaganda region (the Central Kazakhstan). 1,8-cyneol (11.77\%), (4aR,7S, 7aS)-nepetalactone (18.75\%), caryophyllene (3.53\%), germacrene D (5.21\%) are characterized as the main components.

Screening for antimicrobial and analgesic activity of $N$. pannonica essential oil showed the indicated antimicrobial activity against gram-positive test strains of Staphylococcus aureus ATCC 6538 and Bacillus subtilis ATCC 6633, and yeast fungus Candida albicans ATCC 10231, and also demonstrated poor antibacterial activity against the gram-negative test strain Escherichia coli ATCC 25922.

The analgesic activity of $N$. pannonica essential oil is comparable to the diclofenac sodium drug.

\section{References}

1 Павлов Н.В. Флора Казахстана / Н.В. Павлов. — Алма-Ата: Наука, 1964. — Т. 7. — 497 с.

2 Salehi B. Nepeta species: From farm to food applications and phytotherapy / B. Salehi, M. Valussi, A.K. Jugran, M. Martorell, K. Ramírez-Alarcón, Z.Z. Stojanović-Radić, H. Antolak, D. Kręgiel, K.S. Mileski， M. Sharifi-Rad, W.N. Setzerj, M.L. Cádiz-Gurreak, A. Segura-Carreterok, B. Şenerm, J. Sharifi-Rad // Trends in Food Science \& Technology. — 2018. — Vol. 80. — P. 104-122. https://doi.org/10.1016/j.tifs.2018.07.030

3 Afshar A.S. Growth inhibition of human breast cancer cells and down-regulation of ODC1 and ADA genes by Nepeta binaloudensis / A.S. Afshar, F.S. Nematpour, M. Meshkani, A. Khafi // Revista Brasileira De Farmacognosia-Brazilian Journal of Pharmacognosy. — 2017. — Vol. 27, Issue 1. — P. 84-90. https://doi.org/10.1016/J.BJP.2016.07.005

4 Ahmad N. Insecticidal activities and phytochemical screening of crude extracts and its derived fractions from three medicinal plants Nepeta leavigata, Nepeta kurramensis and Rhynchosia reniformis / N. Ahmad, Z.K. Shinwari, J. Hussain, I. Ahmad // Pakistan Journal of Botany. — 2016. - Vol. 48, Issue 6. - P. 2485-2487.

5 Al-Oqail M.M. Anticancer activity of chloroform extract and sub-fractions of Nepeta deflersiana on human breast and lung cancer cells: An in vitro cytotoxicity assessment / M.M. Al-Oqail, E.S. Al-Sheddi, M.A. Siddiqui, J. Musarrat, A.A. Al-Khedhairy, N.N. Farshori // Pharmacognosy Magazine. - 2015. — Vol. 11 (Suppl. 4). — P. 598-605. https://doi.org/10.4103/0973-12961296.172968

6 Al-Taweel A.M. Nepeta deflersiana attenuates isoproterenol-induced myocardial injuries in rats: possible involvement of oxidative stress, apoptosis, inflammation through nuclear factor (NF)-kB down regulation / M.A. Al-Taweel, M. Raish, S. Perveen, G.A. Fawzy, A. Ahmad, M.A., Ansari, M. Shahid, M.A. Ganaie // Phytomedicine. - 2017. — Vol.34. - P. 67-75. https://doi.org/10.1016/j.phymed.2017.08.003

7 Avasthi A.S. Bioassay guided screening, optimization and characterization of antioxidant compounds from high altitude wild edible plants of Ladakh / A.S. Avasthi, M. Bhatnagar, N. Sarkar, S. Kitchlu, S. Ghosal // Journal of Food Science \& Technology. 2016. — Vol. 53(8). - P. 3244-3252. https://doi.org/10.1007/s13197-016-2300-2

8 Bibi T. Ethnomedicinal uses of plants in the treatment of paediatric geohelminth infections in Kalat district of Northern Balochistan / T. Bibi, M. Ahmad, S.E. Edwards, N.M. Tareen, R. Jabeen, I. Abdullah // Pakistan Journal of Ethnopharmacology. — 2016. — Vol. 183. - P. 176-186. https://doi.org/10.1016/j.jep.2016.02.029

9 Rehman T. Investigation of nepetolide as a novel lead compound: antioxidant, antimicrobial, cytotoxic, anticancer, antiinflammatory, analgesic activities and molecular docking evaluation / T. Rehman, A. Khan, A. Abbas, J. Hussain, F.U. Khan, K. Stieglitz, S. Ali // Saudi Pharmaceutical Journal. — 2017. — Vol. 26, Issue 3. - P. 422-429. http://doi.org/10.1016/ j.jsps.2017.12.019.

10 Formisano C. Chemical constituents and biological activities of Nepeta species / C. Formisano, D. Rigano, F. Senatore // Chemistry and Biodiversity. — 2011. — Vol. 8. — P. 1783-1818. https://doi.org/10.1002/cbdv.201000191

11 Sharma A. Phytochemical composition of essential oils isolated from different species of genus Nepeta of Labiatae family: a review / A. Sharma, D.S. Cannoo // Pharmacophore. - 2013. - Vol. 4 (6). - P. 181-211.

12 Sherden N.S. Identification of iridoid synthases from Nepeta species: Iridoid cyclization does not determine nepetalactone stereochemistry / N.H. Sherden, B. Lichman, L. Caputi, D. Zhao, M.O. Kamileen, C.R. Buell, S.E. O'Connora // Phytochemistry. 2018. — № 145. — P. 48-56. https://doi.org/10.1101/179572

13 Курамагомедов М.К. Сравнительный анализ компонентного состава эфирного масла из надземной части Nepeta pannonica L. и Nepeta nepetella L., выращенных в условиях интродукции в Горном Дагестане / М.К. Курамагомедов, А.М. Мусаев, А.М. Алиев, Ф.А. Вагабова, Г.К. Раджабов, З.А. Гусейнова // Естественные и точные науки. — 2017. — Т. 11, № 1. - C. 60-64.

14 Kobaisy M. Composition and Phytotoxic activity of Nepeta pannonica L. essential oil / M. Kobaisy, M. Tellez, F. Dayan, N. Gemejieva // Journal of Essential Oil Research. — 2012. — № 17 (6). — P. 704-707. https://doi.org/10.1080/10412905. 2005.9699037 
15 Хабриев Р.У. Руководство по экспериментальному (доклиническому) изучению новых фармакологических веществ / Р.У. Хабриев. - М.: Медицина, 2005. — 832 с.

16 Государственная фармакопея Республики Казахстан. - Алматы: Жібек жолы, 2008. - Т. 1. — 592 с.

17 Инновационный патент № 23950 от 20.05.2010 г. Эфирное масло Nepeta nuda L. (котовник голый), обладающее антибактериальной активностью, потенциальной анальгетической активностью, аттрактантной активностью для кошачьих и репеллентной активностью против комаров и тараканов / Е.М. Сулейменов.

Г.К. Курмантаева, А.Б. Марченко, С.А. Ивасенко, Р.Б. Сейдахметова, М.К. Смагулов, Г.А. Атажанова

\title{
Nepeta pannonica эфир майының химиялық құрамы және биологиялық белсенділігі
}

\begin{abstract}
Жергілікті флора өсімдіктерінен биологиялық белсенді заттардың жаңа көздерін іздеу қазіргі фитохимиялық ғылымның перспективалы бағыты болып табылады. Мақалада хромато-массспектрометрия әдісімен Қарағанды облысында өсетін мажар көкжалбызынан (Nepeta pannonica) алынған эфир майы үлгілерінің құрамы зерттелген. Өсімдік мүшелеріне байланысты майдың химиялық құрамындағы айырмашылықтар анықталды. Nepeta pannonica-тің эфир майы гидродистилляция әдісімен оқшауланған, олардың химиялық құрамы хромато-масс-спектрометрия әдісімен зерттелген. Талдау үшін эфир майларының компоненттік құрамын анықтаудың бірыңғай әдісі, сондай-ақ 5975 Cinert MSD масс-спектрометриялық детекторы бар 7890A Agilent Technologies хроматографиялық жүйесі қолданылды. Өсімдік гүлдерінің эфир майларындағы мәліметтерге сәйкес мына заттар анықталды: 1,8-цинеол, непетолактон, гермакрен Д. Мажар көкжалбызының эфир майының микробқақарсы және анальгетикалық белсенділігіне скрининг жүргізілді.
\end{abstract}

Kiлm сөздер: Nepeta pannonica, эфир майы, газ хроматографиясы, масс-спектрометрия, химиялық құрамы, микробқақарсы және анальгетикалық белсенділігі.

\section{Г.К. Курмантаева, А.Б. Марченко, С.А. Ивасенко, Р.Б. Сейдахметова, М.К. Смагулов, Г.А. Атажанова \\ Химический состав и биологическая активность эфирного масла Nepeta pannonica}

\begin{abstract}
Поиск новых источников биологически активных веществ из растений местной флоры является перспективным направлением современной фитохимической науки. В статье методом хромато-массспектрометрии изучен состав образцов эфирного масла, выделенных из котовника венгерского (Nepeta pannonica L.), произрастающего в Карагандинской области. Выявлены различия химического состава масла в зависимости от органов растения. Эфирное масло котовника венгерского выделено методом гидродистилляции, его химический состав изучен методом хромато-масс-спектрометрии. Для анализа использовалась унифицированная методика определения компонентного состава эфирных масел, а также хроматографическая система 7890A Agilent Technologies с масс-спектрометрическим детектором 5975 С. Согласно данным, в эфирных маслах цветков растения определены следующие вещества: 1,8-цинеол, непеталактон, гермакрен Д. Проведен скрининг эфирного масла котовника венгерского на антимикробную и анальгетическую активность.
\end{abstract}

Ключевые слова: Nepeta pannonica, эфирное масло, газовая хроматография, масс-спектрометрия, химический состав, антимикробная и анальгетическая активность.

\section{References}

1 Pavlov, N.V. (1964). Flora Kazakhstana [Flora of Kazakhstan]. (Vol. 7). Alma-Ata: Nauka [in Russian].

2 Salehi, B., Valussi, M., Jugran, A.K., Martorell, M., Ramírez-Alarcón, K., \& Stojanović-Radić, Z.Z. et al. (2018). Nepeta species: From farm to food applications and phytotherapy. Trends in Food Science \& Technology, 80, $104-122$. https://doi.org/10.1016/j.tifs.2018.07.030

3 Afshar, A.S., Nematpour, F.S., Meshkani, M., \& Khafi, A. (2017). Growth inhibition of human breast cancer cells and downregulation of ODC1 and ADA genes by Nepeta binaloudensis. Revista Brasileira De Farmacognosia - Brazilian Journal of Pharmacognosy, 27, 1, 84-90. https://doi.org/10.1016/J.BJP.2016.07.005 
4 Ahmad, N., Shinwari, Z.K., Hussain, J., \& Ahmad, I. (2016). Insecticidal activities and phytochemical screening of crude extracts and its derived fractions from three medicinal plants Nepeta leavigata, Nepeta kurramensis and Rhynchosia reniformis. Pakistan Journal of Botany, 48, 6; 2485-2487.

5 Al-Oqail, M. M., Al-Sheddi, E.S., Siddiqui, M.A., Musarrat, J., Al-Khedhairy, A.A., \& Farshori, N.N. (2015). Anticancer activity of chloroform extract and sub-fractions of Nepeta deflersiana on human breast and lung cancer cells: An in vitro cytotoxicity assessment. Pharmacognosy Magazine, 11 (4), 598-605. https://doi.org/10.4103/0973-1296-1296.172968

6 Al-Taweel, A.M., Raish, M., Perveen, S., Fawzy, G.A., Ahmad, A., \& Ansari, M.A. et al. (2017). Nepeta deflersiana attenuates isoproterenol-induced myocardial injuries in rats: possible involvement of oxidative stress, apoptosis, inflammation through nuclear factor (NF)-kB down regulation. Phytomedicine, 34, 67-75. https://doi.org/10.1016/ j.phymed.2017.08.003

7 Avasthi, A.S., Bhatnagar, M., Sarkar, N., Kitchlu, S., \& Ghosal, S. (2016). Bioassay guided screening, optimization and characterization of antioxidant compounds from high altitude wild edible plants of Ladakh. Journal of Food Science \& Technology, 53(8), 3244-3252. https://doi.org/10.1007/s13197-016-2300-2

8 Bibi, T., Ahmad, M., Edwards, S.E., Tareen, N.M., Jabeen, R., \& Abdullah, I. (2016). Ethnomedicinal uses of plants in the treatment of paediatric geohelminth infections in Kalat district of Northern Balochistan. Pakistan Journal of Ethnopharmacology, 183, 176-186. https://doi.org/10.1016/j.jep.2016.02.029

9 Rehman, T., Khan, A., Abbas, A., Hussain, J., Khan, F.U., Stieglitz, K., \& Ali, S. (2017). Investigation of nepetolide as a novel lead compound: antioxidant, antimicrobial, cytotoxic, anticancer, anti-inflammatory, analgesic activities and molecular docking evaluation. Saudi Pharmaceutical Journal, 26, 3, 422-429. http://doi.org/10.1016/j.jsps.2017.12.019.

10 Formisano, C., Rigano, D., \& Senatore, F. (2011). Chemical constituents and biological activities of Nepeta species. Chemistry and Biodiversity, 8, 1783-1818. https://doi.org/10.1002/cbdv.201000191

11 Sharma, A., \& Cannoo, D.S. (2013). Phytochemical composition of essential oils isolated from different species of genus Nepeta of Labiatae family: a review. Pharmacophore, 4 (6), 181-211.

12 Sherden, N.S., Lichman, B., Caputi, L., Zhao, D., Kamileen, M.O., Buell, C. R., \& O'Connora, S.E. (2018). Identification of iridoid synthases from Nepeta species: Iridoid cyclization does not determine nepetalactone stereochemistry. Phytochemistry, 145, 48-56. https://doi.org/10.1101/179572

13 Kuramagomedov, M.K., Musaev, A.M., Aliev, A.M., Vagabova, F.A., Radzhabov, G.K., \& Gusejnova, Z.A. (2017). Sravnitelnyi analiz komponentnogo sostava efirnogo masla iz nadzemnoi chasti Nepeta pannonica L. i Nepeta nepetella L., vyrashchennykh v usloviiakh introduktsii v Gornom Dagestane [Comparative analysis of the component composition of essential oil from the aboveground part of Nepeta pannonica L. and Nepeta nepetella L. grown under the conditions of introduction in Mountain Dagestan]. Estestvennye i tochnye nauki - Natural and Precise Sciences, 11, 1, 60-64 [in Russian].

14 Kobaisy, M., Tellez, M., Dayan, F., \& Gemejieva, N. (2012). Composition and Phytotoxic activity of Nepeta pannonica L. essential oil. Journal of Essential Oil Research, 17 (6), 704-707. https://doi.org/10.1080/10412905.2005.9699037

15 Habriev, R.U. (2005). Rukovodstvo po eksperimentalnomu (doklinicheskomu) izucheniiu novykh farmakologicheskikh veshchestv [Guidelines for the experimental (preclinical) study of new pharmacological substances]. Moscow: Meditsina [in Russian].

16 Gosudarstvennaia farmakopeia Respubliki Kazakhstan [The State Pharmacopoeia of Republic of Kazakhstan]. (Vol. 1). (2008). Almaty: Zhibek zholy [in Russian].

17 Suleimenov, E.M. (2010). Innovatsionnyi patent № 23950 ot 20.05.2010. Efirnoe maslo Nepeta nuda L. (kotovnik golyi), obladaiushchee antibakterialnoi aktivnostiu, potentsialnoi analgeticheskoi aktivnostiu, attraktantnoi aktivnostiu dlia koshachikh $i$ repellentnoi aktivnostiu protiv komarov i tarakanov [Innovative patent No. 23950 from 20.05.2010. Nepeta nuda L. essential oil (bare pot), having antibacterial activity, potential analgesic activity, attractant activity for cats and repellent activity against mosquitoes and cockroaches] [in Russian]. 